\title{
Variabilidad genética mediante AFLP en tres relictos de Gomortega keule (Molina) Baillon, especie endémica chilena en peligro de extinción
}

\section{Genetic diversity revealed by AFLP in three relicts of Gomortega keule (Molina) Baillon, endemic and endangered Chilean species}

\author{
Catherine Delaveau ${ }^{1 *}$, Glenda Fuentes-Arce ${ }^{2}$, Eduardo Ruiz ${ }^{2}$, Rodrigo Hasbún ${ }^{1}$, Matilde Uribe $^{1}$, \\ Sofía VALENZUELA ${ }^{1,3}$ \\ ${ }^{1}$ Facultad de Ciencias Forestales y Centro de Biotecnología, Universidad de Concepción, Casilla 160-C, Universidad de \\ Concepción, Concepción, Chile. \\ 2Departamento de Botánica, Facultad de Ciencias Naturales y Oceanográficas, Universidad de Concepción, Concepción, \\ Chile. \\ ${ }^{3}$ Genómica Forestal S.A., Centro de Biotecnología, Universidad de Concepción, Concepción, Chile. \\ *catherine.delaveau@gmail.com
}

\begin{abstract}
RESUMEN
Gomortega keule, especie arbórea endémica de Chile y única representante de la familia Gomortegaceae, se encuentra catalogada en peligro de extinción y despierta gran interés científico por su restringida área de distribución. El objetivo de este trabajo fue estimar la variabilidad genética de 24 individuos de $G$. keule ubicados en tres relictos de la provincia de Concepción, mediante marcadores AFLP (polimorfismos en la longitud de los fragmentos amplificados) para futuros estudios de conservación. Se probaron 16 combinaciones de partidores y se eligieron las tres combinaciones más informativas para detectar diferencias entre individuos. Estas combinaciones generaron un total de 156 fragmentos, siendo un $62 \%$ de ellos polimórficos. El sitio con mayor porcentaje de polimorfismos correspondió a Cerro Neuque, con un 84,6 $\%$, seguido por Coroney y Hualqui con 59,6 y 42,3\%, respectivamente. El análisis de varianza molecular detectó un $73 \%$ de variación dentro de las poblaciones y un $27 \%$ de variación entre ellas. El estudio muestra que la técnica de AFLP puede ser utilizada en $G$. keule de forma rápida y confiable para identificar poblaciones remanentes y apoyar su conservación tanto ex situ como in situ.
\end{abstract}

Palabras clave: Gomortegaceae, Laurales, marcadores moleculares, loci polimórfico.

\begin{abstract}
Gomortega keule is an endemic woody species to Chile and the only member of the Gomortegaceae family. It is currently an endangered species and is of great scientific interest due to its small area of distribution. The aim of this research was to estimate genetic diversity of 24 individuals of $G$. keule selected from three isolated relict populations from the province of Concepción using AFLP (Amplified fragment length polymorphism) for future conservation issue. Sixteen combinations of primers for AFLP were tested and three combinations of them were selected as the most informative, revealing differences between individuals. These primer combinations generated 156 fragments, of which $62 \%$ were polymorphic. The relict population with the highest percentage of polymorphisms was located in Cerro Neuque with 84.6 $\%$, followed by Coroney and Hualqui with 59.6 and $42.3 \%$, respectively. The analysis of molecular variance detected 73 $\%$ variation within populations and $27 \%$ variation amoung populations. This study supported that AFLP can be used in G. keule as a fast and reliable test to identified remaining populations of the species. Thus, this technique can be used to support ex situ or in situ conservation of G. keule.
\end{abstract}

KEYwoRDS: Gomortegaceae, Laurales, molecular markers, polymorphic loci. 


\section{INTRODUCCIÓN}

Gomortega keule (Molina) Baillon es una especie arbórea en estado crítico de conservación (González 1998) que habita en áreas pequeñas y fragmentadas de la Cordillera de la Costa entre Cauquenes $35^{\circ} 46^{\prime} \mathrm{S}$ y Arauco $37^{\circ} 41^{\prime} \mathrm{S}$ (Hechenleitner et al. 2005). Gomortega pertenece a la familia Gomortegaceae, la cual es endémica y monotípica (Rodríguez et al. 2005). Se reportan sólo 22 poblaciones insertas en plantaciones exóticas, lo que las mantiene en constante amenaza (San Martín \& Sánchez 1999, Rodríguez \& Quezada 2001). Además de los problemas de distribución, la regeneración de la especie se ve afectada por factores como depredación de semillas por roedores, colecta de frutos por los lugareños y apertura del bosque, así como también por la baja capacidad germinativa de la semilla y el lento crecimiento durante los primeros años (Donoso \& Escobar 1985, Le Quesne \& Stark 2006).

Los antecedentes disponibles concluyen que la especie se estaría propagando, principalmente, por vía asexual, al observarse escasa o nula regeneración sexual en terreno (Villegas et al. 2003).

La utilización de marcadores moleculares es una herramienta útil en numerosos estudios de diversidad genética, aportando información para desarrollar planes de conservación en especies amenazadas. Algunas de las técnicas empleadas son: polimorfismo del ADN amplificado al azar (RAPD) (Torres et al. 2003), intermicrosatélites (ISSR) (Huang et al. 2009), microsatélites (Azpilicueta et al. 2004) y AFLP (Wen-Kun et al. 2008). En específico, esta última técnica ha demostrado ser exitosa por su reproducibilidad, además de detectar un gran número de fragmentos polimórficos sin requerir conocimiento previo de la secuencia de ADN de la especie en estudio (Mueller \& Wolfenbarger 1999, Meudt \& Clarke 2007).

En G. keule, la diversidad genética fue estimada en 33 individuos de poblaciones del rango norte de su distribución mediante ISSR (Herrera et al. 2005). Posteriormente García-Gonzáles et al. (2008) ampliaron el estudio a su rango sur utilizando la misma técnica en 11 poblaciones. En tanto Lander et al. (2007) aislaron y caracterizaron ocho microsatélites sin realizar estudios de variabilidad genética.

Considerando el estado de conservación de G. keule y la falta de información de su presencia en algunos sectores de su rango de distribución, el objetivo de este estudio fue estimar la variabilidad genética de $G$. keule mediante marcadores AFLP en tres relictos de la provincia de Concepción con el fin de contribuir a futuras propuestas de conservación ex situ de la especie.

\section{MATERIALES Y MÉTODOS}

Material Vegetal

En tres relictos de G. keule ubicados en la provincia de
Concepción se colectaron hojas en pleno crecimiento. Se muestrearon 8 individuos en cada relicto, los cuales se encontraban a más de $3 \mathrm{~m}$ de distancia, fueron identificados con GPS (Global Positioning System) y denominados: 1) Cerro Neuque ( $\left.36^{\circ} 34^{\prime} \mathrm{S} ; 7^{\circ} 54^{\prime} \mathrm{O}\right)$, 2) Coroney ( $36^{\circ} 40^{\prime} \mathrm{S}$; $72^{\circ} 51^{\prime}$ O) y 3 ) Hualqui ( $36^{\circ} 53^{\prime} \mathrm{S} ; 7^{\circ} 55^{\prime}$ O) (Fig. 1). El material fue almacenado a $-80^{\circ} \mathrm{C}$ hasta su análisis.

\section{EXTRACCIÓN ADN}

Se aisló ADN utilizando el protocolo de extracción de ADN para plantas DNeasy Plant Mini (QIAGEN). Se utilizaron $100 \mathrm{mg}$ de tejido vegetal, el que fue homogeneizado con un equipo de lisis mecánica, adicionándole microesferas de cerámica a una velocidad de $6 \mathrm{~m} \mathrm{~s}^{-1}$ durante $15 \mathrm{~s}$.

El ADN extraído se cuantificó por espectrofotometría de absorbancia a una longitud de onda de $260 \mathrm{~nm}$ (A260). Usando este método también se obtuvo una estimación de la pureza del $\mathrm{ADN}$ por medio de las relaciones de absorbancia (A260/A280) y (A260/A230).

Para evaluar la integridad del ADN extraído se mezclaron alícuotas de $5 \mu \mathrm{L}$ con $1 \mu \mathrm{L}$ de tampón de carga (6X DNA loading dye, Fermentas) y se analizó mediante electroforesis a $60 \mathrm{~V}$ durante $30 \mathrm{~min}$ en gel de agarosa al 1 $\%(\mathrm{p} / \mathrm{v})$. La visualización del ADN en los geles se realizó usando un transiluminador y digitalización mediante fotografía digital.

\section{AFLP}

La técnica de AFLP se desarrolló según el protocolo publicado por Hasbún et al. (2012). Éste está basado en el método descrito por Vos et al. (1995), pero utilizando fluoróforos adheridos a los partidores de la amplificación selectiva, con el fin de ser separados por electroforesis capilar y reconocidos con un secuenciador automático.

La técnica consta de tres pasos: 1) digestión- ligación: se realizó la digestión a $10 \mathrm{ng} \mu \mathrm{L}^{-1}$ de $\mathrm{ADN}$ de G. keule con las enzimas EcoRI y MseI, posteriormente se ligó el producto de la digestión con adaptadores de doble cadena, específicos para cada enzima, 2) amplificación preselectiva: con el producto del paso anterior se realizó una reacción en cadena de la polimerasa (PCR) utilizando partidores del tipo EcoRI + AC y $M s e I+C, 3)$ amplificación selectiva: se probaron 16 combinaciones de partidores selectivos del tipo EcoRI+3 / MseI+3 o +4 marcados con fluoróforos.

Para realizar el análisis genético de todos los individuos se procedió primero a elegir las combinaciones más informativas. Esta selección se realizó según los siguientes criterios; capacidad de amplificación, cantidad de fragmentos polimórficos (entre 50 y $500 \mathrm{pb}$ ) y nitidez de banda. El producto de amplificación se visualizó en un gel de agarosa al $1 \%(\mathrm{p} / \mathrm{v})$ con $2 \mu \mathrm{L}$ de bromuro de etidio, posteriormente se realizó la electroforesis capilar con el secuenciador automático ABI PRISM 3130xl Genetic Analyzer. 

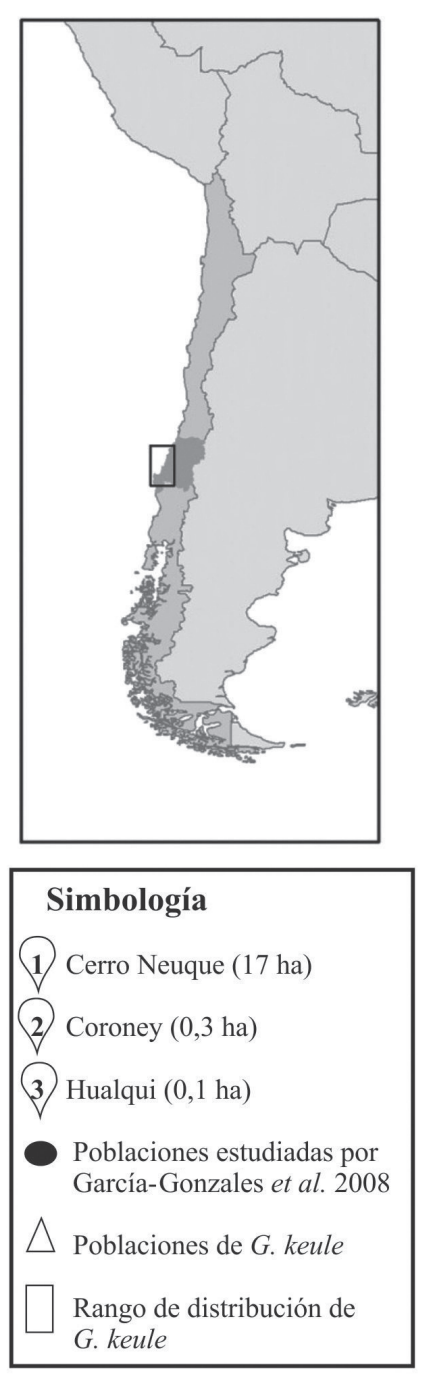

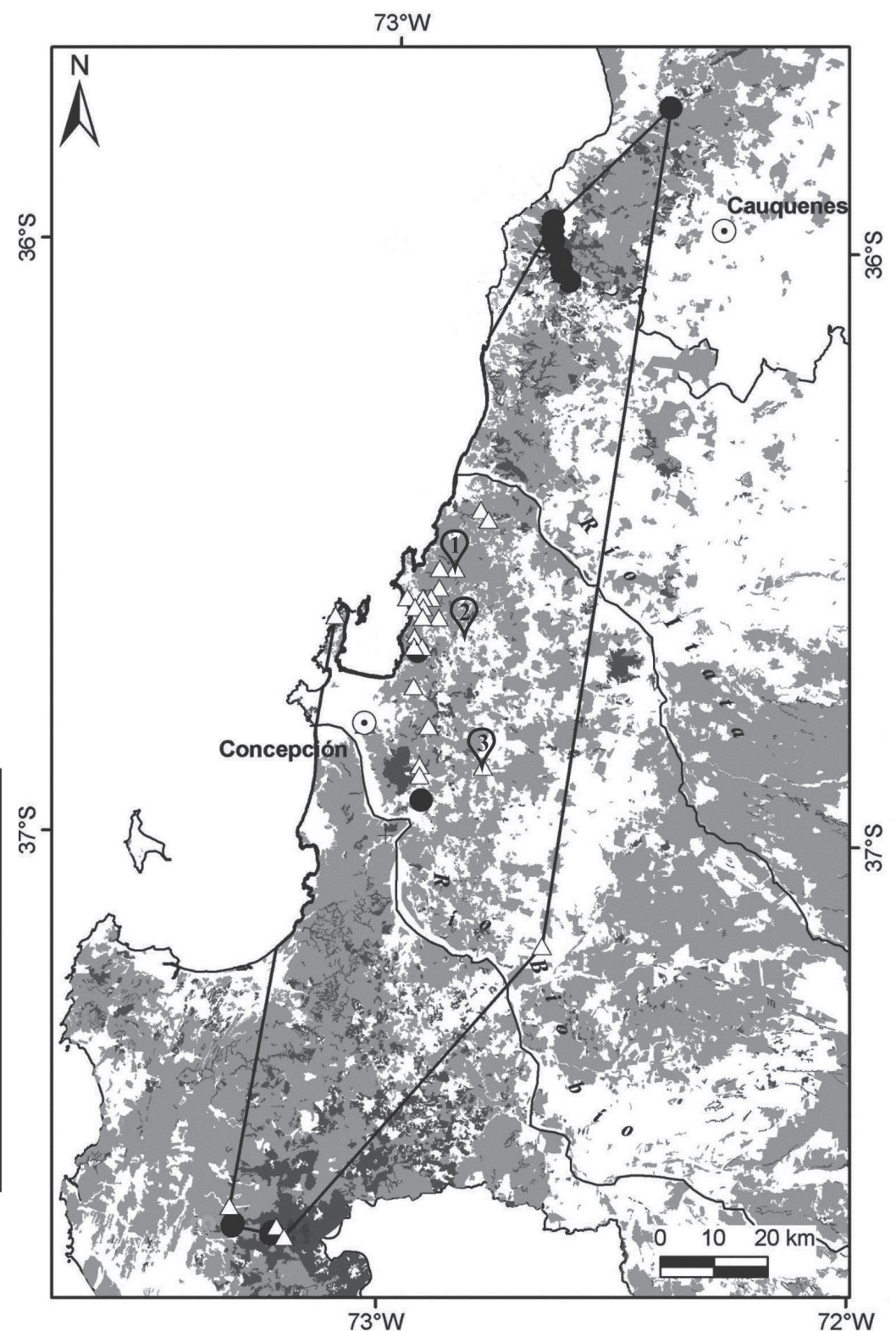

Figura 1. Localización de los relictos de G. keule estudiados y rango de distribución de la especie. Mapa modificado de García-Gonzales et al. 2008.

FIGURE 1. Locating relict populations of G. keule studied and distribution range of the species. Modified map from García-Gonzales et al. 2008.

Los datos fueron analizados utilizando el software GeneMapper v4.0 con el cual se confeccionó una matriz binaria $(0 / 1)$ representando la ausencia o presencia del fragmento.

\section{AnÁlisis de Datos}

A partir de la matriz binaria y utilizando el programa GenAlEx versión 6.1 (Peakall y Smouse 2006), se calcularon los siguientes índices de variabilidad genética: porcentaje de loci polimórficos $(\% \mathrm{P})$, heterocigosidad esperada $(\mathrm{He})$ e índice de Shannon (S). La estructuración genética se estimó a través de un análisis de varianza molecular (AMOVA) (Excoffier et al. 1992) y se realizó una prueba de Mantel para correlacionar valores de distancia genética y distancia geográfica.

\section{RESULTADOS}

De las 16 combinaciones de partidores AFLP evaluadas en $G$. keule, se seleccionaron tres combinaciones que permitieron visualizar bandas nítidas con más de 12 fragmentos 
polimórficos cada una (Tabla I). Con ellas se obtuvo un total de 156 fragmentos de ADN y de los cuales el $62 \%$ fue polimórfico. Las combinaciones restantes no fueron consideradas por no amplificar fragmentos en la especie o generar un patrón de fragmentos poco reproducible y con escasos polimorfismos.

La combinación EcoRI+ACA / MseI+CAT presentó el mayor porcentaje de polimorfismo arrojando 39 fragmentos polimórficos que corresponden al 68,42 \% del total de fragmentos detectados para esta combinación.
A su vez, con $E c o$ RI+ACA /MseI+CCG y EcoRI+ACC / $M s e \mathrm{I}+\mathrm{CAT}$ se detectaron un 58,8 y $41,6 \%$ de polimorfismo, respectivamente.

Para el análisis genético, con el total de fragmentos obtenidos con las tres combinaciones de partidores, los índices de diversidad genética detectados por AFLP en los relictos de G. keule indican que el mayor porcentaje de loci polimórficos se observó en individuos de Cerro Neuque, con un $84,6 \%$ de polimorfismo seguido por Coroney, con $59,6 \%$, y Hualqui, con $42,3 \%$ de polimorfismo (Tabla II).

TABLA I. Porcentaje de polimorfismo detectado en 24 individuos de G. keule con tres combinaciones selectivas de marcadores AFLP (polimorfismos en la longitud de los fragmentos amplificados).

TABLE I. Polymorphic percentage in 24 G. keule individuals detected with three selective AFLP (Amplified fragment length polymorphism) primer sequences.

\begin{tabular}{|c|c|c|c|}
\hline \multirow{2}{*}{$\begin{array}{l}\text { COMBINACIÓN } \\
\text { SELECTIVA* }\end{array}$} & \multicolumn{2}{|c|}{$\mathrm{N}^{\mathrm{o}}$ Fragmentos } & \multirow{2}{*}{ \% DE POLIMORFÍSMO } \\
\hline & TOTAL & PoLIMÓRFICOS & \\
\hline $\begin{array}{l}E c o \mathrm{RI}+\mathrm{ACA} / \\
M s e \mathrm{I}+\mathrm{CAT}\end{array}$ & 57 & 39 & 68,42 \\
\hline $\begin{array}{l}E c o \mathrm{RI}+\mathrm{ACC} / \\
M s e \mathrm{I}+\mathrm{CAT}\end{array}$ & 48 & 28 & 41,66 \\
\hline $\begin{array}{l}E c o \mathrm{RI}+\mathrm{ACA} / \\
M s e \mathrm{I}+\mathrm{CCG}\end{array}$ & 51 & 30 & 58,82 \\
\hline Total & 156 & 97 & - \\
\hline Promedio & 52 & - & - \\
\hline
\end{tabular}

*A, C, G, T representan los nucleótidos adenina, citosina, guanina, timina / A, C, G, T simbolize nucleotides adenine, guanine, cytosine, thymine.

TABLA II. Índices de diversidad genética detectados en tres relictos de G. keule utilizando marcadores AFLP (polimorfismos en la longitud de los fragmentos amplificados).

TABLE II. Genetic diversity index detected with AFLP (Amplified fragment length polymorphism) marker in three G. keule relict.

\begin{tabular}{l|c|c|c|c}
\hline ReLicto & $\begin{array}{c}\text { Fragmentos } \\
\text { POLIMÓRFICOS }\end{array}$ & $\begin{array}{c}\text { P } \\
(\%)\end{array}$ & S & He \\
\hline Coroney & 31 & 59,62 & $0,30(0,03)$ & $0,20(0,02)$ \\
Hualqui & 22 & 42,31 & $0,19(0,03)$ & $0,12(0,02)$ \\
Cerro Neuque & 44 & 84,62 & $0,46(0,03)$ & $0,31(0,02)$ \\
Promedio & & 62,18 & $0,32(0,02)$ & $0,21(0,01)$ \\
\hline
\end{tabular}

P: porcentaje de polimorfismo; S: índice de Shannon; He: heterocigosidad esperada. Valores entre paréntesis indican error estándar / P: polymorphic percentage; S: Shannon index; He: expected heterozygosity. Values in parentheses represent standard error. 
El mayor porcentaje de la variación detectado por el análisis de varianza molecular se registró dentro de las poblaciones con un $73 \%$, mientras que entre los tres relictos de $G$. keule se registró el $27 \%$ restante. El grado de estructuración genética entre las poblaciones de G. keule medido por el coeficiente de diferenciación genética $(F \mathrm{st})$ fue 0,267 , indicando que las poblaciones se encuentran altamente diferenciadas. Además, al realizar la prueba de Mantel no se obtuvo una correlación entre valores de distancia genética y distancia geográfica $\left(\mathrm{R}^{2}=0,691 ; \mathrm{p}=\right.$ 0,160).

Cabe indicar que sólo en Coroney se observó regeneración sexual; sin embargo, en visitas posteriores se pudo constatar la nula supervivencia de las plántulas.

\section{DISCUSIÓN}

Al comparar los resultados obtenidos en $G$. keule con otras Lauraceae y en categoría de amenaza, mediante marcadores dominantes, tales como RAPD en Neolitsea sericeae $(\mathrm{P}=$ $50,5 \%$, Wang et al. 2005) y AFLP en Litsea szemaois (P $=80,79 \%$ y ISSR, $\mathrm{P}=87,01 \%$, Ci et al. 2008) se puede ordenar a $G$. keule en un rango intermedio. Por otro lado, si se consideran los valores de diversidad genética reportados por Hamrick \& Godt (1989) para especies leñosas longevas $(\mathrm{P}=49,3 \%, \mathrm{He}=0,148), G$. keule presenta mayores niveles de diversidad $(\mathrm{P}=62,18 \%, \mathrm{He}=0,212)$. No obstante, estos valores de diversidad genética se asemejan a los obtenidos para especies endémicas $(\mathrm{He}=0,2)$ por medio de RAPD (Nybom 2004).

Lander et al. (2009, 2010) determinaron que la polinización de la especie es entomófila y el polen de $G$. keule se mueve hasta $6 \mathrm{~km}$ desde pequeños parches o árboles aislados hasta sitios de mayor tamaño y viceversa, siendo los primeros fundamentales en la conectividad del flujo génico. Esto se ve reflejado en los valores obtenidos en la prueba de Mantel $\left(\mathrm{R}^{2}=0,691 ; \mathrm{p}=0,160\right)$, los cuales concuerdan a los reportados por García-Gonzales et al. (2008) $(\mathrm{r}=-0,000 ; \mathrm{p}=0,72)$ que señalan que las poblaciones no presentan aislamiento por distancia.

Adicionalmente, al comparar el índice de Shannon promedio para $G$. keule $(\mathrm{S}=0,322)$ con una especie endémica longeva, tal como Araucaria araucana (Molina) K. Koch ( $\mathrm{S}=0,65$, Bekessy et al. 2002), se observa que la variabilidad genética de $G$. keule es menor. Esto puede deberse a la dispersión entomófila del polen de G. keule, ya que especies cuyo polen es dispersado anemófilamente, como es el caso de $A$. araucana, presentan mayor diversidad genética (Hamrick \& Godt 1996).

Estudios realizados por Herrera et al. (2005) en 33 individuos de $G$. keule y utilizando ISSR reportaron valores de variación entre y dentro de la población (30 y
$70 \%$ respectivamente). García-Gonzales et al. (2008) por su parte, a través del mismo marcador molecular y en 223 individuos de $G$. keule obtuvieron valores idénticos a los de este estudio ( 27 y $73 \%$ ), los cuales son los esperados para especies perennes leñosas y de polinización cruzada (Hamrick et al. 1992).

El nivel de diferenciación genética $(F \mathrm{st}=0,267)$ determinado para $G$. keule en este estudio es similar al informado por García-Gonzales et al. (2008) ( $F \mathrm{st}=0,275)$ siendo ambos valores mayores al descrito para especies longevas, perennes y de polinización cruzada $(F \mathrm{st}=0,094)$ (Hamrick \& Godt 1996). La limitada dispersión de la semilla puede ser la responsable de este resultado, ya que las semillas suelen encontrarse intactas bajo el dosel y a pocos metros del árbol. Según Villegas et al. (2003) y García-Gonzales et al. (2008), al encontrarse los relictos de G. keule rodeados por plantaciones exóticas se favorece la pérdida de agentes dispersores (del polen y la semilla).

Los tres relictos estudiados, al igual que otras poblaciones de G. keule, se encuentran aislados e insertos en plantaciones de pino, reproduciéndose sólo vegetativamente. Durante el período de colecta de material se observó reiterada intervención antrópica como el control de malezas con el fin de evitar incendios forestales, esto genera daño al eliminar plántulas y evitar la regeneración natural de la especie. A pesar de las condiciones en las cuales se encuentra G. keule en los sitios de estudio se obtuvo una mayor variabilidad genética en los relictos de mayor superficie (Fig.1). Según Hamrick \& Godt (1996) los factores que más influyen sobre la variabilidad genética entre las poblaciones son el número de individuos y la superficie. Esto se corrobora en este trabajo dado que se utilizó la misma técnica e igual número de individuos para todos los relictos y sólo la superficie fue distinta.

En este estudio se reafirma que los AFLP son una poderosa herramienta para estudios de genética de poblaciones, ya que con sólo 3 combinaciones selectivas de AFLP se estimó la variabilidad de G. keule en los relictos analizados. Resultados similares se lograron en otras especies amenazadas utilizando tres o cuatro combinaciones de AFLP (Palacios et al. 1999, Ni et al. 2006, Ci et al. 2008). A su vez empleando ISSR en $G$. keule se obtiene una estructuración genética similar a la obtenida en este estudio (Herrera et al. 2005, García-Gonzáles et al. 2008).

Adicionalmente, la variabilidad genética presentada en los relictos contribuye a ampliar los antecedentes en la especie, ya que reportes anteriores y de zonas cercanas al área de estudio presentan menores niveles de variabilidad. Por ejemplo, Penco $(\mathrm{P}=67,95 \%, \mathrm{~S}=0,3708$, GarcíaGonzales et al. 2008) en comparación con Cerro Neuque $(\mathrm{P}=84,62 \%, \mathrm{~S}=0,46)$.

Por lo tanto, es posible estimar la variabilidad genética en las poblaciones naturales remanentes de G. keule y detectar a 
futuro variantes genéticas únicas y/o centros de variabilidad genética con el fin de contribuir en estudios enfocados a la conservación ex situ de esta especie amenazada.

\section{AGRADECIMIENTOS}

Los autores agradecen al proyecto DIUC No 209.142.0281.0 de la Dirección de Investigación de la Universidad de Concepción por el financiamiento y a Genómica Forestal S.A. por la cooperación en este estudio. Catherine Delaveau agradece a Conicyt por su beca doctoral.

\section{BIBLIOGRAFÍA}

Azpilicueta, M., H. Caron, C. Bodénes \& L. Gallo. 2004. SSR markers for analysing South American Nothofagus species. Silvae Genetica 53(5-6): 240-243.

Bekessy, S., T. Allnutt, A. Premoli, A. Lara, R. Ennos, M. Burgman, M. Cortés \& A. Newton. 2002. Genetic variation in the vulnerable and endemic Monkey Puzzle tree, detected using RAPDs. Heredity 88: 243-249.

Ci ,X., J. ChEn, Q. Li \& J. Li. 2008. AFLP and ISSR analysis reveals high genetic variation and inter-population differentiation in fragmented populations of the endangered Litsea szemaois (Lauraceae) from Southwest China. Plant Systematic and Evolution 273: 237-246.

Donoso, C. \& B. Escobar. 1985. Germinación de Gomortega keule (Mol.) Baillon. Bosque 6(2): 120-122.

Excoffier, L., P. Smouse \& J. Quattro. 1992. Analysis of molecular variance inferred from metric distances among DNA haplotypes: application to human mitochondrial DNA restriction data. Genetics 131: 479-491.

García-Gonzales, R., B. Carrasco, P. Peñailillo, L. Letelier, R. Herrera, B. Lavandero, M. Moya \& P. Caligari. 2008. Genetic variability and structure of Gomortega keule (Molina) Baillon (Gomortegaceae) relict populations: geographical and genetic fragmentation and its implications for conservation. Botany 86: 1299-1310.

GonzÁlez, M. 1998. Gomortega keule. IUCN Red List of Threatened Species. Version 2012.2. URL: http://www. iucnredlist.org/details/31357/0 Viewed: march 03, 2013.

Hamrick, J. \& M. GodT. 1989. Allozyme diversity in plant species. In: A.H.D. Brown, M.T. Cregg, A.L. Kahler \& B.S. Weir (eds.), Plant population genetics, breeding and genetic resources. pp. 43-63. Sinauer, Sunderland, MA.

Hamrick, L. \& M. GodT. 1996. Effects of life history traits on genetic diversity in plant species. Philosophical Transactions of the Royal Society Biological Sciences 351: 1291-1298.

Hamrick, J., M. Godt \& S. Sherman-Broyles. 1992. Factors influencing genetic diversity in woody plant species. New Forests 6: 95-124.

Hasbún, R., C. Iturra, P. Moraga, P. Wachtendorff, P. Quiroga \& S. Valenzuela. 2012. An efficient and reproducible for production of AFLP markers in tree genomes using fluorescent capillary detection. Tree Genetics \& Genome
8(4): 925-931.

Hechenleitner, P., M. Gardner, P. Thomas, C. Echeverría, B. Escobar, P. Brownless \& C. Martínez. 2005. Plantas amenazadas del Centro-Sur de Chile. Distribución, conservación y propagación. Primera Edición. pp. 74-75. Universidad Austral de Chile y Real Jardín Botánico de Edimburgo.

Herrera, R., M. Arias, M. Moya-León, P. Peñailillo \& M. Wilkinson. 2005. Genetics variation in a Chilean endangered endemic: Gomortega keule (Molina) Baillon. Biodiversity and Conservation 14: 2871-2881.

Huang, Y., C. Zhang \& D. Li. 2009. Low genetic diversity and high genetic differentiation in the critically endangered Omphalogramma souliei (Primulaceae): implications for its conservation. Journal of Systematics and Evolution 47(2): 103-109.

Lander, T., D. Boshier \& S. Harris. 2007. Isolation and characterization of eight polymorphic microsatellite loci for the endangered, endemic Chilean tree Gomortega keule (Gomortegaceae). Molecular Ecology Notes 7: 1332-1334.

LANder, T., D. Boshier \& S. Harris. 2009. Flower and fruit production and insect pollination of the endangered chilean tree, Gomortega keule in native forest, exotic pine plantation and agricultural environments. Revista Chilena de Historia Natural 82(3): 403-412.

Lander, T., D. Boshier \& S. Harris. 2010. Fragmented but not isolated: Contribution of single trees, small patches and long-distance pollen flow to genetic connectivity for Gomortega keule, an endangered Chilean tree. Biological Conservation 143(11): 2583-2590.

Le Quesne, C. \& D. StaRk. 2006. Latifoliadas chilenas: Gomortega keule (Mol.) Baillon. En: C. Donoso (ed.), Las especies arbóreas de los bosques templados de Chile y Argentina. Autoecología. pp. 277-284. Valdivia, Chile. Marisa Cuneo Ediciones.

Meudt, H. \& A. Clarke. 2007. Almost forgotten or latest practice AFLP aplications, analyses and advances. Plant Science 12(3): 106-117.

Mueller, U. \& L. Wolfenbarger. 1999. AFLP genotyping and fingerprinting. Trends in Ecology and Evolution 14: 389394.

Ni, X., Y. Huang, L. Wu, R. Zhou, S. Deng, D. Wu, B. Wang, G. Su, T. TANG \& S. SHI. 2006. Genetic diversity of the endangered Chinese endemic herb Primulina tabacum (Gesneriaceae) revealed by amplified length polymorphism (AFLP). Genetica 127: 177-183.

Nyвом, H. 2004. Comparison of different nuclear DNA markers for estimating intraspecific genetic diversity in plants. Molecular Ecology 13: 1143-1155.

Palacios, C., S. Kresovich \& F. González-Candelas. 1999. A Population genetic study of the endangered plant species Limonium dufourii (Plumbaginaceae) based on amplified fragment length polymorphism (AFLP). Molecular Ecology 8: 645-657.

Peakall, R. \& P. Smouse. 2006. GENALEX6: genetic analysis in Excel. Population genetic software for teaching and research. Molecular Ecology Notes 6: 288-295.

Rodríguez, R., \& M. Quezada. 2001. Gomortegaceae. En: C. Marticorena \& R. Rodríguez (eds.), Flora de Chile, Vol. 2(1).Winteraceae-Ranunculaceae. Ediciones Universidad 
de Concepción, Chile. 16-18.

Rodríguez, R., E. Ruiz \& J. Elissetche. 2005. Árboles en Chile. Ed. Universidad de Concepción, Chile. 183 pp.

SAn Martín, J. \& A. SÁnchez. 1999. Las comunidades relictas de Gomortega keule (Gomortegaceae, Magnoliopsida) en Chile Central. Anales Jardín Botánico Madrid 57(2): 317326.

Torres, E., J. Iriondo \& C. Pérez. 2003. Genetic structure of an endangered plant, Antirrhinum microphyllum (Scrophulariaceae): allozyme and RAPD analysis. American Journal of Botany 90(1): 85-92.

Villegas, P., C. Le Quesne \& CH. LusK. 2003. Estructura y dinámica de una población de Gomortega keule (Mol.) Baillon en un rodal antiguo de bosque valdiviano, Cordillera de
Nahuelbuta, Chile. Gayana Botánica 60(2): 107-113.

Vos, P., R. Hogers, M. Bleeker, M. Reijans, T. van de Lee, M. Hornes, A. Frijters, J. Pot, J. Peleman, M. Kuiper \& M. ZABEAU. 1995. AFLP: a new technique for DNA fingerprinting. Nucleic Acids Research 23(21): 44074414.

Wang, Z.S., S.Q. An, H. Liu, X. Leng, J.W. Zheng \& Y.H. Liu. 2005. Genetic structure of the endangered plant Neolitsea sericea (Lauraceae) from the Zhoushan Archipelago using RAPD markers. Annals of Botany 95: 305-313.

Wen-Kun, H., G. Jian-Ying, W. Fang-HaO. G. Bi-Da \& X. BingYAN. 2008. AFLP analyses on genetic diversity and structure of Eupatorium adenophorum populations in China. Chinese Journal of Agricultural Biotechnology 5 (1): 33-41.

Recibido: 08.03.13

Aceptado: 30.05 .13 